\title{
Development of a Robust and Quantitative High Throughput Screening Method for Antibiotic Production in Bacterial Libraries
}

\author{
Elizabeth M. Murray ${ }^{\dagger}$, Catherine F. Allen ${ }^{\dagger}$, Tess E. Handy ${ }^{\dagger}$, Clair A. Huffine ${ }^{\dagger, \ddagger}$, Whitney R. Craig ${ }^{\ddagger}$, Sarah
} C. Seaton $\$$, Amanda L. Wolfe ${ }^{*}$

${ }^{\dagger}$ Department of Biology and $¥$ Department of Chemistry, University of North Carolina Asheville, One University Heights, Asheville, North Carolina, 28804, United States

sIndigo Ag, 500 Rutherford Avenue, Boston, Massachusetts, 02129, United States

\section{Supporting Information}

\section{Supporting Figure}

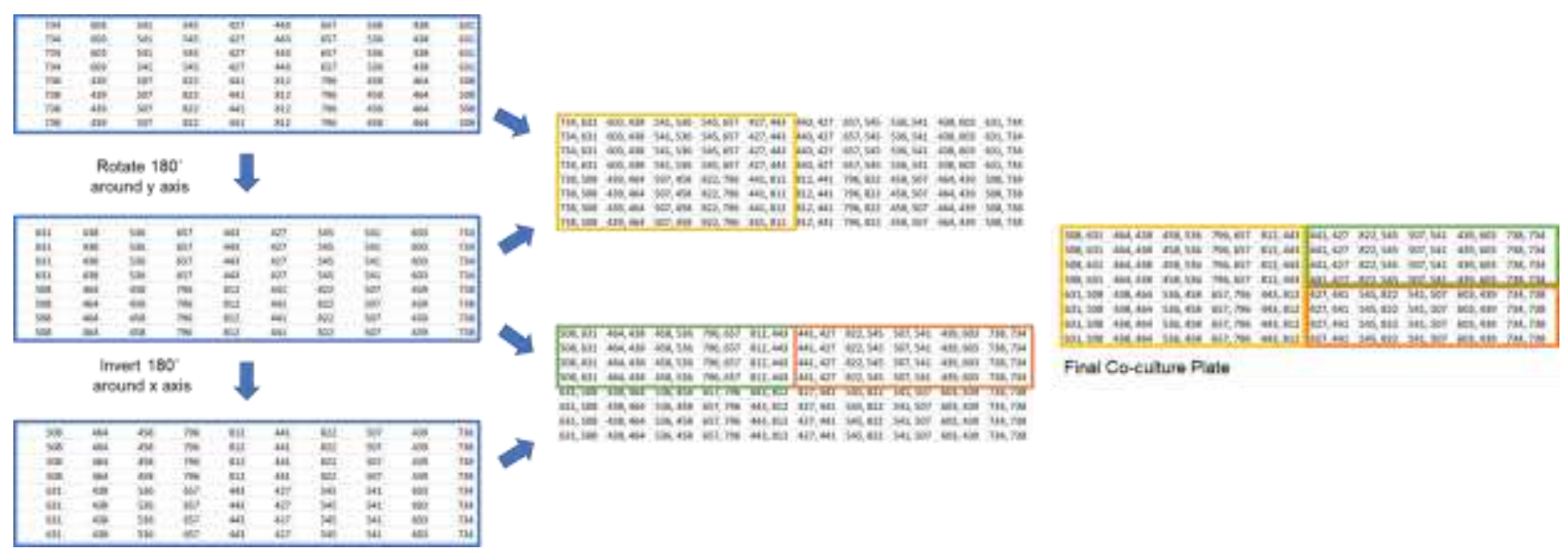

Figure S1. Process of flipping the 96 well VWR $®$ tissue culture plate $180^{\circ}$ along both the $x$ and $y$ axes to create a final co-culture plate of unique pairs.

\section{Data Procession}

To create a baseline, the averaged value of $32.5 \%$ TSB without pathogen is subtracted from each well. The new well values eliminate any absorbance created by the media.

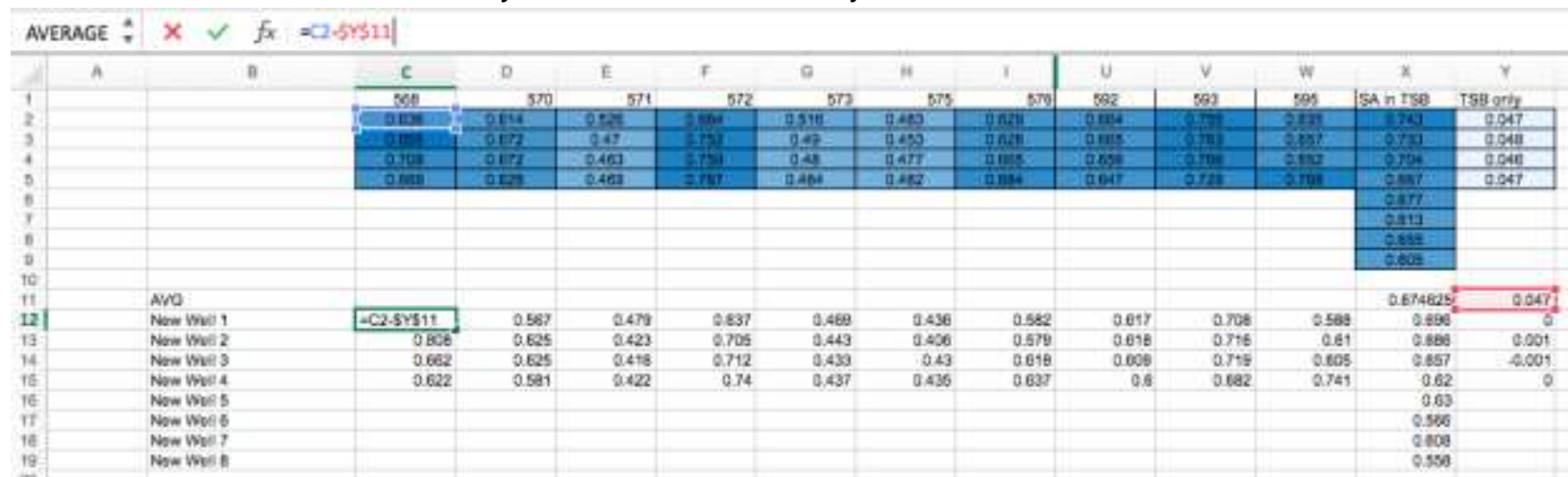

Figure S2. Layout of data normalization from subtracting the average of TSB only (32.5\% TSB) from each well absorbance values.

The ratio of pathogen inhibition is found by dividing each well by the new well average of $32.5 \%$ TSB with pathogen. The resulting ratios represent the amount of pathogen inhibition in each well. 


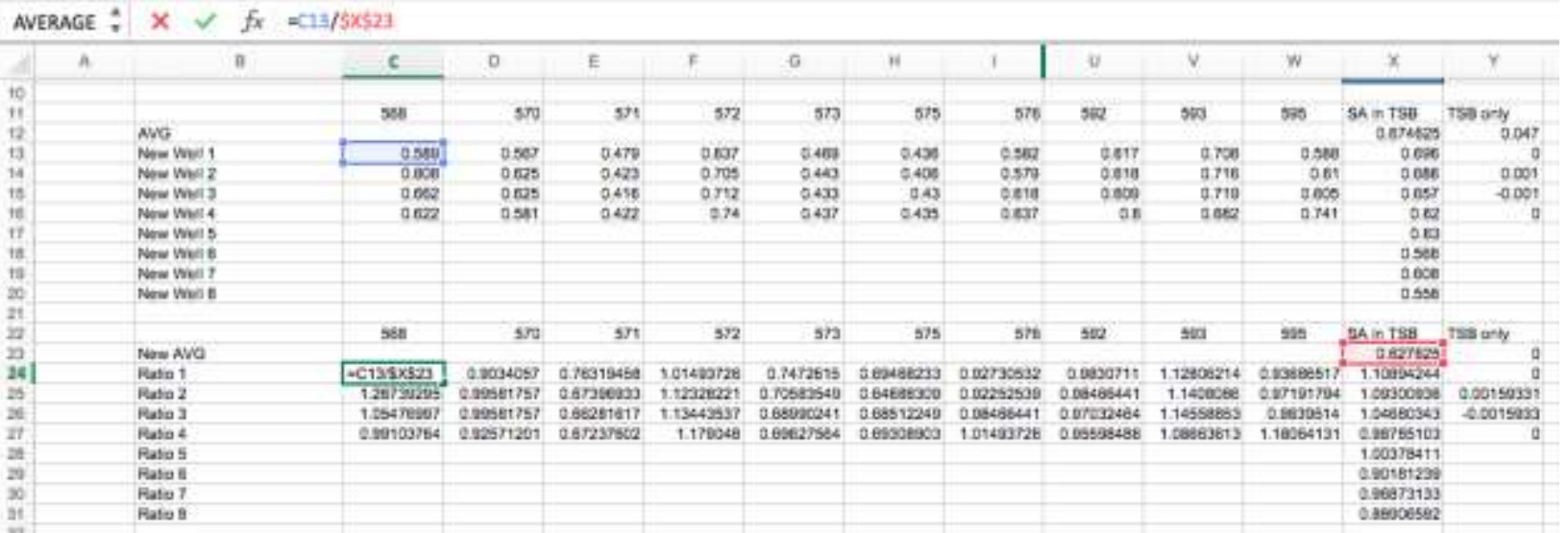

Figure S3. Layout of inhibition ratio calculation.

The ratios for each bacteria are averaged and the standard deviation is found. To make a $95 \%$ confidence interval the standard deviation is multiplied by two and then added onto the ratio average. Producers are identified if the resulting value is below 1 . Strong producers are identified if their ratio average is also below 0.7 , meaning there was at least $30 \%$ inhibition. Producers are highlighted in yellow, while strong producers are highlighted in orange.

\begin{tabular}{|c|c|c|c|c|c|c|c|c|c|c|c|c|c|c|}
\hline \multicolumn{2}{|c|}{ AVERAGE : } & \multicolumn{13}{|c|}{$x \vee f_{x} \quad=$ C34*5A535+C33 } \\
\hline & A & e & c & 0 & E & $F$ & 6 & H & 1 & $u$ & $v$ & w & $x$ & y \\
\hline स्म & & & 596 & 90 & sता & $9 \pi$ & 573 & 575 & 578 & Fक्य & 523 & 59 & 54 in 138 & 75 anty. \\
\hline 23 & & Now AN/O & & & & & & & & & & & I02TES & a \\
\hline 74 & & Rato I & Q85sised? & satoses & 47. & 101283720 & प 74rats:s & प $62 \times 6033$ & a & 0 ogsumा & $1,1200 \mathrm{e}$ /4 & 2.20stos:17 & 1 10earzes & d \\
\hline 28 & & Rata 2 & 1 atractus & azosen17s? & to traseass & 1.02120221 & ancosisto & D EAESESC? & a mz2525210 & $0.004054-1$ & 11450000 & SEryaire4 & $1,5000005 t$ & ( toblsurar \\
\hline$x$ & & Ratan 3 & 1 sstreqa? & c9ase175? & qabzets17 & 1.13443937 & Q 680000041 & 0 coss12240 & O.984as441 & Oefrated & 1 1Assaes? & obous14 & 100060349 & 2 \& 201523 \\
\hline 27 & & Basin 6 & de sancares & อ928rt201 & aerzarana & 1.170048 & to eanzrsad & atempess & 101692128 & ODS\$as4as & 1.taec3613 & 1,10566131 & a 206786103 & a \\
\hline$a$ & & Matins & & & & & & & & & & & 1 9urra411 & \\
\hline$\approx$ & & Ratio is & & & & & & & & & & & a solst2an & \\
\hline$x^{2}$ & & Rata $t$ & & & & & & & & & & & a searats & \\
\hline tit & & Ratioll & & & & & & & & & & & esenobses? & \\
\hline 12 & & & & & & & & & & & & & & \\
\hline xa & & Rata AvO & $1867 n+45$ & a ass 10821 & 0.00050303 & t.112azs? & e. & 0.67034623 & o sezase1 & 0.87351125 & 1.12527305 & 151336906 & 1 & a \\
\hline 36 & & STUEV & (15) & 0.04719:3s & cosesesea & 0.06022114 & a 025 atsos & 0.02243930 & D. O4SOCont9 & 0.01333087 & 5.easpres & 2.11253614 & G58060451 & 1. $0.00+30089$ \\
\hline 35 ? & & 6 so & tes3 & 1.5078599 & 276rues? & 12521790 & a 7814355 & 4. $224+22$ & t DSZ3reate & 10001724 & 117487314 & 12394163 & 1. 161 rese? & 2 a.0026018d \\
\hline 36 & & & & & & & & & & & & & & \\
\hline at & & & ses & 500 & $57 t$ & 572 & 573 & 575 & 576 & 822 & 503 & 305 & SA in TSB & 189 any \\
\hline 34 & & Rato Avo & 1.86791478 & 9.95513a21 & U Va9300303 & t.t12ases? & O. Nostar6 & O. Breaceza & o sezase! & o. & 1.123273 as & 1.01334390 & & 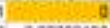 \\
\hline 39 & & Non Froduser Meas Farye & 230770653 & $20956857 \mathrm{a}$ & Q. 08950167 & a. $\div 3025428$ & 0.05161269 & 0.04496796 & 0.09501836 & o.02seet1c & 2.053506es & 22250720 & 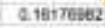 & $20.0026018 d$ \\
\hline 40 & & Procuser Moun farges & & & & & & & & & & & & \\
\hline 41 & & Limit of Aocteplance & 1 & $\therefore$ & t & t & 1 & 1 & 1 & 1 & 1 & 1 & 1 & 1 \\
\hline$A_{2}$ & & arong Produces & a.7 & 0.7 & Q.7. & 07 & 07 & a) & 0.7 & a7 & 27 & 27 & 2.7 & 0.7 \\
\hline
\end{tabular}

Figure S4. Layout of data calculation for producer identification.

Table S1. Activity and genus level sequencing of bacterial library. Key: $=$ activity not determined; $1=$ statistically significant ( $95 \%$ confidence) inhibition of pathogen compared to control; $2=\geq 30 \%$ inhibition of pathogen.

\begin{tabular}{lccc}
\hline $\begin{array}{l}\text { Sample } \\
\text { Number: }\end{array}$ & $\begin{array}{l}\text { E. coli } \\
\text { active }\end{array}$ & $\begin{array}{c}\text { S. aureus } \\
\text { active }\end{array}$ & Genus-level Sequencing Results: \\
& & & \\
\hline RGRF B10 & & 2 & Pseudomonas
\end{tabular}




\begin{tabular}{|c|c|c|c|}
\hline SS 422 & - & 1 & Pseudomonas \\
\hline SS 439 & 2 & 0 & Bacillus, Brevibacterium \\
\hline SS 449 & 0 & 1 & Chromobacterium \\
\hline SS 454 & - & 1 & Chromobacterium \\
\hline SS 519 & 2 & 2 & Brevibacillus \\
\hline SS 528 & 2 & 2 & Pseudomonas \\
\hline SS 560 & 0 & 1 & Pseudomonas \\
\hline SS 581 & 0 & 2 & Collimonas \\
\hline SS 614 & _ & 1 & Herbaspirillum \\
\hline SS 616 & _ & 2 & Pseudomonas \\
\hline SS 617 & _ & 1 & Pseudomonas \\
\hline SS 619 & _ & 2 & Pseudomonas \\
\hline SS 623 & _ & 1 & Pseudomonas \\
\hline SS 625 & 0 & 2 & Pseudomonas \\
\hline SS 626 & _ & 1 & Pseudomonas \\
\hline SS 636 & _ & 2 & Pseudomonas \\
\hline SS 650 & _ & 1 & Pseudomonas \\
\hline SS 651 & _ & 1 & Kluyvera, Leclercia, Enterobacter \\
\hline SS 654 & 2 & - & Serratia \\
\hline
\end{tabular}




\begin{tabular}{|c|c|c|c|}
\hline SS 655 & 2 & - & Serratia \\
\hline SS 659 & 2 & 2 & Serratia \\
\hline SS 660 & 2 & 1 & Serratia \\
\hline SS 661 & _ & 2 & Pseudomonas \\
\hline SS 662 & _ & 2 & Pseudomonas \\
\hline SS 672 & 2 & _- & Serratia \\
\hline SS 674 & 2 & - & Serratia \\
\hline SS 682 & 2 & - & Serratia \\
\hline SS 692 & - & 1 & $\begin{array}{l}\text { Cedecea, Enterobacter, Raoultella, } \\
\text { Lelliottia, Klugvera }\end{array}$ \\
\hline SS 701 & 2 & 1 & Herbaspirillum \\
\hline SS 722 & 1 & _ & Microbacterium \\
\hline SS 724 & 0 & 2 & Pseudomonas \\
\hline SS 729 & 1 & 1 & Bacillus \\
\hline SS 730 & _ & 1 & Serratia \\
\hline SS 751 & 1 & 0 & Streptomyces \\
\hline SS 752 & 1 & 0 & Bacillus \\
\hline SS 764 & 0 & 2 & Bacillus \\
\hline SS 779 & 1 & 2 & Bacillus, Brevibacterium \\
\hline
\end{tabular}




\begin{tabular}{llll} 
SS 783 & 1 & 0 & Streptomyces \\
SS 792 & 0 & 1 & Streptomyces \\
SS 794 & - & 1 & Bacillus, Brevibacterium \\
SS 821 & 1 & 0 & Staphylococcus \\
SS 827B & - & 2 & Pseudomonas \\
\hline
\end{tabular}

